\title{
Research Paper: Investigating the Effect of Safety Training on the Safety Climate of Employees Working in Kermanshah Province, Iran Oil Refining Company
}

\author{
Mohammad Reza Omidi ${ }^{*}$ (D), Meysam Jafari Eskandari² (D, Nabi Omidi² (iD)
}

1. Department of Industrial Engineering, Faculty of Industrial Engineering, South Tehran Branch, Islamic Azad University, Tehran, Iran. 2. Department of Industrial Engineering, Payame Noor University, Tehran, Iran.

\begin{tabular}{|l|l|l|}
\hline $\begin{array}{c}\text { Use yur device to scan } \\
\text { and read the article online }\end{array}$ & $\begin{array}{l}\text { Citation: Omidi MR, Jafari Eskandari M, Omidi N. Investigating the Effect of Safety Training on the Safety Climate of Employees } \\
\text { Working in Kermanshah Province, Iran Oil Refining Company. Health in Emergencies and Disasters Quarterly. 2020; 6(1):23-28. http:// } \\
\text { dx.doi.org/10.32598/hdq.6.1.222.11 }\end{array}$ \\
doi: $:$ http://dx.doi.org/10.32598/hdq.6.1.222.11
\end{tabular}

\section{(i) (3)}

Article info:

Received: 09 Jun 2020

Accepted: 01 Sep 2020

Available Online: 01 Oct 2020

\section{Keywords:}

Safety, Employee health, Accidents

\section{ABSTRACT}

Background: To achieve a safe and healthy environment for human beings and the dynamic industry as a critical factor in sustainable development, it is necessary to deal with accidents and eliminate their underlying causes radically. Safety climate shows the attitude and general perception of an organization and its management towards safety. This study aimed to investigate the effect of safety training on the safety climate of employees working in Kermanshah Province (Iran) Oil Refining Company.

Materials and Methods: The present study was quasi-experimental interventional research conducted in 2019 in Kermanshah Province, Iran Oil Refining Company, with a pre-test, posttest design. The study population included all employees working in different departments of Kermanshah Oil Refining Company. Sampling was done randomly with 240 people. The questionnaire used in the present study had the demographic information questionnaire. To assess the workers' climate safety, the safety climate questionnaire of the British Safety Organization was used. The level of reliability was 0.84 . Information coding was performed in SPSS V. 23, and the Mean \pm SD of demographic information and atmosphere scores were determined. To investigate the effect of interventions on the safety climate before and one month after educational and technical interventions, a pairwise comparison was used.

Results: The comparison of the mean scores of the safety climate components before the intervention was equal to 2.61 and after the educational intervention reached 3.72. All components of the safety climate increased after the intervention. The highest mean increase was related to the component of "safety communications" with 1.74 units. The lowest increase was associated with the component of "employee intervention in safety" with 0.68 units. Before and after the intervention, "safety enhancement" had the highest score among the safety climate components. Before the intervention, the lowest mean score was related to the "safety violations" component, which after the intervention, the "licensing system" had the lowest mean score.

Conclusion: The educational intervention improved the safety climate in Kermanshah Oil Refining Company. Therefore, safety training plays an essential role in increasing the oil and gas industry's safety climate.

\section{* Corresponding Author:




\section{Introduction}

I

$\mathrm{n}$ the last two decades, Iran has developed in several areas. It has experienced rapid industrial and economic growth, which has led to economic and social changes with its good and bad consequences [1]. One of the unfavorable effects of industrialization is the increasing number and variety of occupational accidents. Besides damaging human resources, occupational accidents cause high economic costs [2]. The oil and gas industries are among the largest job-creating sectors in Iran. Safety issues related to the oil and gas industries have always been a significant problem and primary concern in many countries. The leading causes of accidents and occupational injuries in the oil and gas industries are human error and unsafe practices due to lack of safety programs [3]. Without adequate awareness and risk identification, even the best safety programs will not be successful [4].

Today, industry executives have realized that one of the most important ways to reduce work-related accidents is to pay attention to safety. Safety climate is a way to measure workers' perceptions of security in the work environment over a while. In other words, it emphasizes employees' shared understanding of policies, procedures, practices, the overall importance, and real priority of safety in the work environment, which leads to changes in safety improvement [5]. Research on the effect of psychological and organizational factors on behavioral risks and the possibility of injury in the work environment has shown that the safety climate significantly impacts these factors $[6,7]$.

The term "safety climate" was coined by Zohar in 1980. He defined the safety climate as the "employees' shared perceptions of the policies, procedures, and practices relating to safety such as the overall importance and real priority of safety in the work environment" [8].

Safety climate is a particular form of organizational climate rooted in individual perceptions of the importance of safety in the work environment and guides employees' actions and workgroups and personal attitudes toward work [9]. Safety climate as a subset of safety culture examines employees' perceptions and inferences about the work environment, management's level of interest in safety and safety-related measures, and the degree of participation in risk control. Workers' perceptions of safety in the work environment directly correlate with occupational accident rates [10]. Employees who find their job relatively more dangerous are less likely to be involved in accidents. Employees who perceive their work environment as safe also report lower work-related anxiety and stress [11]. Measuring the safety climate has many advantages, as reliable and credible safety measurements can overcome the limitations of traditional and old-fashioned safety measurement methods such as "accident lost time rates" or "accident reporting review" [6].

The limitations of older methods of measuring safety are as follows: 1. Older methods are not so much concerned about providing useful safety-related information for a particular location; 2. They do not offer a tool for assessing employees' exposure to risk; and 3. They are always retrospective, but measuring the safety climate can provide useful information about safety problems before they occur [12]. Kermanshah Oil Refining Company is one of the most experienced oil refining companies in the country, which was put into operation in 1922. During the last 50 years, significant changes have been made in this refinery's materials, processes, and activities. These equipment and procedures have been the scene of accidents more than ever. In this industrial unit, a total of 138 accidents occurred in 2019, in which "burns" and "falls from a height" had the highest percentage of accidents among others.

Studies on the structure of the safety climate in organizations indicate that due to the lack of well-organized training on occupational safety and health in the industry, most workers are not aware of the dangers in their work environment [13]. Researchers believe that safety training is one of the most important factors influencing the safety climate in organizations. Exercise promotes awareness and motivation, followed by a change in attitude, and promotes a safety climate [14]. Considering the importance of safety climate, the study, as one of the factors involved in preventing and reducing occupational accidents and its positive effect, was conducted to evaluate safety training on improving safety climate in Kermanshah Oil Refining Company.

\section{Materials and Methods}

As quasi-experimental interventional research, the study was conducted in 2019 in Kermanshah Oil Refining Company with a pre-test, post-test design. Its purpose was to investigate the effect of safety training on employees' safety climate. The study population included 630 people working in different sections of Kermanshah Oil Refining Company. Using Cochran's formula, a sample of 240 people was selected by random sampling. The sampling process was performed to determine the workers in each section, and they were included in the study as participants. The target population was selected based on the fact that all staff had received safety training. The 
target group faced a variety of hazards and accidents that are common in many work environments. It should be noted that the subjects entered this study with full knowledge of the objectives of the research and they completed the consent form and were assured that the information remained confidential with the researchers and their answers would not cause any job problems for them.

The questionnaire used in the study consisted of two separate parts: the first part related to the background information of the workers (age, marital status, education, work experience, job category, employment status, and previous accident history) and the general information (questionnaire code for each worker, date, and time of completing the questionnaire). In the second part, to assess workers' awareness about the safety climate, the UK safety atmosphere questionnaire of the British safety organization was used. The questionnaire included 43 questions consisting of 11 components of safety climate: management commitment, awareness, and knowledge of safety regulations, communication in the field of safety, violation of safety rules, improvement of safety rules and regulations, the impact of employee intervention on safety, licensing system, encouragement for safety, participation in safety improvement, and risk perception [6].

The questions were scored on a 5-point Likert scale with a maximum score of 5 (strongly agree) to a minimum score of 1 (strongly disagree). Despite the validation of the original version of this questionnaire in Iran, the Persian translation of the questionnaire was tested again for validity and reliability in the present study. To evaluate the questionnaire's reliability, the internal consistency method was calculated by the Cronbach's alpha of 0.84 using SPSS. Besides, to assess the questionnaire's validity, the experts' corrective opinions were collected and applied in the questionnaire.

Performing educational and technical interventions consisted of two stages: the first stage included educational intervention, and the second stage included technological intervention and the repetition of educational intervention. Before the intervention, a briefing session was held for safety experts from all sectors to conduct training classes for workers. In this session, training pamphlets, booklets, instructions, videos, photos, and PowerPoints were presented to the experts. The content of the training course was prepared based on the investigation of accidents and their causes, risk assessment, risks in each job category, and interviews (for surveys of experts and workers). These issues included harmful factors and dangerous conditions in the work environment; empowering the workers on how to deal with hazardous factors and conditions in the work environment, types of personal protective equipment and how to use them properly, workers' awareness of safe and unsafe behaviors and the impact of changing dangerous behaviors and the benefits of safe behaviors, empowering workers to detect unsafe behaviors, workers' understanding of the losses caused by unsuitable workstations and how to set up a suitable workstation in the work environment, the need for short breaks between work, and encouraging workers to take responsibility.

Before starting each training session, workers were interviewed for 15 minutes in a briefing session about the study's objectives and the confidentiality of the information reported by them. In almost all sessions, workers verbally expressed their satisfaction with these sessions. The workers were then divided into 6 groups of 40 people (each group consisting of people from all occupations). The educational interventions consisted of two stages. The interventions were performed in 12 two-hour sessions over 6 weeks (each week on two different days) in each step. Using a computer system, a large monitor, and a training board in this training, workers were involved in group discussions and training. They were surveyed about safe behaviors and how to improve the situation. Also, they were encouraged to address the raised issues.

In this study, the questionnaires were collected in two stages: the first stage before the interventions, the second stage one month after the first training course for data analysis. First, the Mean \pm SD values of demographic information and safety climate scores were determined. After collecting the data, they were entered in SPSS V. 23. First, the Mean \pm SD values of demographic details and safety climate scores were determined. To investigate the effect of interventions on the safety climate before and one month after educational and technical interventions, a pairwise comparison was used.

In this study, the authors observed all ethical considerations about the subjects. All research items were monitored and regulated based on the Helsinki Declaration and the 26 codes of the country's research ethics.

\section{Results}

Out of 240 participants in the study, $24(10 \%)$ were female, and $228(90 \%)$ were male. The Mean \pm SD age of male workers was $39 \pm 4.12$ years, and the Mean \pm SD age of female workers was $34 \pm 0.24$ years. The Mean \pm SD work experience of male and female workers was $13 \pm 2.11$ and $8 \pm 1.11$, respectively. In terms of education level, a total of 25 people $(10.4 \%)$ had diplomas 
and lower education, a total of 142 people $(59.1 \%)$ had bachelor's degrees, and 73 people (30.4\%) had master's degrees and higher education. The Mean \pm SD scores of the safety climate components before and after the educational intervention are presented in Table 1.

They calculated the mean scores of the safety climate components before the intervention, which was equal to 2.61, and after the educational intervention reached 3.72. All components of the safety climate increased after the intervention. The highest increase in the mean component was related to "safety communication", with an increase of 1.74 units. The lowest increase was associated with the component of "staff intervention in safety", with an increase of 0.68 units. Before and after the intervention, "safety enhancement" had the highest score among the safety climate components. Before the intervention, the lowest mean score was related to the "violation of safety rules" component, and after the intervention, the "licensing system" had the lowest mean score.

\section{Discussion}

Lack of attention to safety in industry has caused many injuries and casualties. The purpose of this study was to assess the level of safety climate among employees of Kermanshah Oil Refining Company and the effect of training on improving this vital component. The present study results confirmed that the safety climate among employees working in Kermanshah Oil Refining Company was intermediate (score 2.61 out of 5) before the educational intervention. This indicates a gap in the existing industry to increase the safety atmosphere, and safety items are not used properly. In a study that examined the safety climate level among copper production unit employees, the safety climate level was moderate [15]. Measurement of the Chinese industries' safety climate revealed that China's oil and gas industry's safety climate was low [7]. Aliabadi et al. assessed the country's sugar industry's safety climate and safety culture as weak [16].

Severe et al. assessed the safety climate among workers in various oil and gas industries in European countries as appropriate [9]. Dahl examined the prevailing safety climate among employees in multiple sectors. The results indicated that the safety climate in Asian countries was moderate [3]. In a study, Khandan et al. examined safety factors in various industries in Qom Province, Iran and did not consider the future safety climate in these industries appropriately [17].

In the study of Khomar et al., the safety climate of patients in hospitals and rehabilitation centers in Tehran City, Iran was not assessed as favorable. Considering the presence of safety climate components in this study

Table 1. Mean \pm SD scores of safety climate components before and after the educational intervention

\begin{tabular}{ccc}
\hline \multirow{2}{*}{ Safety Climate Component } & \multicolumn{2}{c}{ Mean \pm SD } \\
\cline { 2 - 3 } & Values Before the Intervention & Values After the Intervention \\
\hline Safety enhancement & $3.37 \pm 0.158$ & $4.12 \pm 0.245$ \\
\hline Production pressure & $3.24 \pm 0.242$ & $4.11 \pm 0.124$ \\
\hline Safety training & $3.12 \pm 0.257$ & $4.07 \pm 0.312$ \\
\hline Management commitment & $2.76 \pm 0.657$ & $3.81 \pm 0.311$ \\
\hline The role of the protection committee & $2.71 \pm 0.612$ & $4.14 \pm 0.325$ \\
\hline Staff intervention in safety & $2.54 \pm 0.571$ & $3.22 \pm 0.312$ \\
\hline Encouragement for safety & $2.31 \pm 0.511$ & $3.71 \pm 0.411$ \\
\hline Safety rules and regulations & $2.27 \pm 0.125$ & $3.72 \pm 0.347$ \\
\hline Licensing system & $2.26 \pm 0.251$ & $3.11 \pm 0.157$ \\
\hline Safety communication & $2.12 \pm 0.148$ & $3.86 \pm 0.169$ \\
\hline Violation of safety rules & $2.07 \pm 0.135$ & $3.12 \pm 0.187$ \\
\hline Total mean & $2.61 \pm 0.514$ & $3.72 \pm 0.672$ \\
\hline
\end{tabular}


showed that the element of "safety enhancement" before the intervention and after the intervention had a higher mean score than other safety climate components among employees. This finding confirmed that employees were eager for the progress made in safety, and employees welcomed the use of advanced and modern safety tools.

In Aliabadi et al. study, among the safety climate components, the element of "management commitment" had a better score than others [16]. Similarly, in Chahak et al. study and Khammar et al. study, the component of "management commitment" had the highest mean score $[18,19]$. Kongsvik et al. showed that "safety enhancement" was more evident among the safety climate components among the oil and gas industry employees [20]. The present study revealed that training improves the safety climate, which confirms the importance of education in increasing safety levels in various industries. In line with the current study results, Grasoo et al. and Bamel et al. studies confirmed that educational intervention had a positive effect on safety performance [5, 21]. The results of Jafari et al. investigation revealed that educational intervention significantly increased the mean scores of "management commitment", "safety training", "safety communications", "staff intervention in safety", "licensing system", "safety rules and regulations", and "violation of safety rules" [12].

\section{Conclusion}

This study confirmed that educational intervention increased and improved the safety climate among Kermanshah Oil Refining Company employees. Therefore, safety training can positively affect expanding the industry's safety climate, ultimately reducing injuries and casualties in accidents at work. If the activity is carried out according to the correct principles and careful planning, it can help promote workers' and society's safety. According to the available statistics on work-related accidents and the resulting personal and social consequences, the importance of educating people about safety could be understood.

Therefore, industries must prevent work accidents by adopting proper safety and health measures through useful and practical training. According to our study results, holding training classes for workers and encouraging group discussion, and expressing the importance of safety in the work environment and technical measures, can have a positive effect on workers' safety behavior and promote organizational culture, and improve safety climate factors. Discussing and sharing people's expe- riences, developing a training program, and spending enough time can also encourage safe behaviors.

One of the study's limitations was the absence of the control group survey due to the company's existing conditions. To overcome this limitation, one month of the intervention was evaluated to examine the higher reliability changes.

\section{Ethical Considerations}

\section{Compliance with ethical guidelines}

All ethical principles are considered in this article. The participants were informed of the purpose of the research and its implementation stages.

\section{Funding}

This research did not receive any grant from funding agencies in the public, commercial, or non-profit sectors.

\section{Authors' contributions}

All authors equally contributed to preparing this article.

\section{Conflict of interest}

The authors declared no conflict of interest.

\section{References}

[1] Asgari H, Omidi MR, Omidi N. Use of econometric techniques to estimate the traumatic trend of road accidents. Journal of Safety Promotion and Injury Prevention. 2019; 6(4):173-82. https://www.sid.ir/En/Journal/ViewPaper.aspx?ID=718061

[2] Omidi MR, Jafari Eskandari M, Raissi S, Shojaei AA. Providing an appropriate prediction model for traffic accidents: A case study on accidents in Golestan, Mazandaran, Guilan, and Ardebil Provinces. Health in Emergencies and Disasters Quarterly. 2019; 4(3):165-72. [DOI:10.32598/hdq.4.3.165]

[3] Dahl Ø, Kongsvik T. Safety climate and mindful safety practices in the oil and gas industry. Journal of Safety Research. 2018; 64:29-36. [DOI:10.1016/j.jsr.2017.12.009] [PMID]

[4] Omidi N, Omidi MR. Estimating accident-related traumatic injury rate by future studies models in semnan province, Iran. Health in Emergencies and Disasters Quarterly. 2018; 3(4):191-8. [DOI:10.32598/hdq.3.4.191]

[5] Grasso M. Towards a broader climate ethics: Confronting the oil industry with morally relevant facts. Energy Research \& Social Science. 2020; 62:101383. [DOI:10.1016/j.erss.2019.101383] 
[6] Nordborg FM, Jones RJ, Oelgemöller M, Negri AP. The effects of ultraviolet radiation and climate on oil toxicity to coral reef organisms: A review. Science of The Total Environment. 2020; 720:137486. [DOI:10.1016/j.scitotenv.2020.137486] [PMID]

[7] Tong R, Yang X, Parker T, Zhang B, Wang Q. Exploration of relationships between safety performance and unsafe behavior in the Chinese oil industry. Journal of Loss Prevention in the Process Industries. 2020; 66:104167. [DOI:10.1016/j. jlp.2020.104167]

[8] Tang KHD, Md Dawal SZ, Olugu EU. A review of the offshore oil and gas safety indices. Safety Science. 2018; 109:344-52. [DOI:10.1016/j.ssci.2018.06.018]

[9] Kvalheim SA, Dahl Ø. Safety compliance and safety climate: A repeated cross-sectional study in the oil and gas industry. Journal of Safety Research. 2016; 59:33-41. [DOI:10.1016/j. jsr.2016.10.006] [PMID]

[10] Probst TM, Goldenhar LM, Byrd JL, Betit E. The Safety Climate Assessment Tool (S-CAT): A rubric-based approach to measuring construction safety climate. Journal of Safety Research. 2019; 69:43-51. [DOI:10.1016/j.jsr.2019.02.004] [PMID]

[11] Khandan M, Vosoughi S, Azrah K, Poursadeghiyan M, Khammar A. Decision making models and human factors: TOPSIS and Ergonomic Behaviors (TOPSIS-EB). Management Science Letters. 2017; 7:111-8. [DOI:10.5267/j.msl.2016.11.008]

[12] Jafari MJ, Gharari M, Kalantari S, Omidi L, Ghaffari M, Asadolah Fardi GR. [The influence of safety training on improvement in safety climate in construction sites of a firm (Persian)]. 2015; 2(4):257-64. https://doi.org/10.22037/ meipm.v2i4.8386

[13] Niv Y, Chorev N, Tal Y, Manor O, Broda A, Peer T, et al. [Culture of safety in the hospital]. Harefuah. 2019; 158(8):520-2. [PMID]

[14] Dunstan E, Cook JL, Coyer F. Safety culture in intensive care internationally and in Australia: A narrative review of the literature. Australian Critical Care. 2019; 32(6):524-39. [DOI:10.1016/j.aucc.2018.11.003] [PMID]

[15] Khaleghinejad A, Ziaaldini M. Relationship between employees' safety climate and safety performance with respect to mediating effect of safety knowledge and safety motivation in Sarcheshmeh copper complex. Journal of Health and Safety at Work. 2015; 5(4):69-86. https:/ / www.sciencedirect. com/science/article/abs/pii/S1036731418301711

[16] Mirzaie Aliabadi M, Feize Arefi M, Gholami S, Chahardooli Z, Rostami F. Investigating safety climate and its structural dimensions in a sugar industry using Nordic Questionnaire (NOSACQ-50). Journal of Occupational and Environmental Health. 2017; 3(1):59-67. http://oeh.arums.ac.ir/files/site1/ user_files_767397/godadmin-A-10-2-42-cbef129.pdf

[17] Khandan M, Aligol MH, Shamsi M, Poursadeghiyan M, Biglari H, Koohpaei A. Occupational health, safety, and ergonomics challenges and opportunities based on the organizational structure analysis: A case study in the selected manufacturing industries in Qom Province, Iran, 2015. Annals of Tropical Medicine and Public Health. 2017; 10(3):606-11. https:/ / www.atmph.org/article.asp?issn=1755-6783;year=201 7 ; volume $=10 ;$ issue $=3$; spage $=606$; epage $=611$; aulast $=$ Khandan

[18] Khammar A, Poursadeghiyan M, Marioryad H, Nabi Amjad R, Alimohammadi M, Khandan M. Patient safety climate and its affecting factors among rehabilitation health care staff of hos- pitals and rehabilitation centers in Iran, Tehran. Iranian Rehabilitation Journal. 2019; 17(1):39-48. [DOI:10.32598/irj.17.1.39]

[19] Firoozi Chahak A, Barkhordarei Firozabadei A, Mirzaei Alavijeh $M$. [Assessment of safety climate in one of the mine in Yazd Province (Persian)]. Tolooebehdasht. 2014; 13(4):164-72. http://tbj.ssu.ac.ir/article-1-1203-en.html

[20] Kongsvik T, Dahl Ø, Holmen IM, Thorvaldsen T. Safety climate and health complaints in the Norwegian aquaculture industry. International Journal of Industrial Ergonomics. 2019; 74:102874. [DOI:10.1016/j.ergon.2019.102874]

[21] Bamel UK, Pandey R, Gupta A. Safety climate: Systematic literature network analysis of 38 years (1980-2018) of research. Accident Analysis \& Prevention. 2020; 135:105387. [DOI:10.1016/j.aap.2019.105387] [PMID] 